\title{
Structure of the Handbook
}

The Handbook of Proximity Relations is organized in four parts, respectively devoted to the analytical dimensions of the notion of proximity, to the methods and analytical tools, to the dimensions of innovation and knowledge, and finally to the emerging issues and challenges to this approach. They are preceded and followed by an Introduction and a general Conclusion.

The introduction by André Torre and Delphine Gallaud, entitled "Proximity relations in the 21st century", is a general assessment of the theoretical approaches of proximity relations and their possible developments. The authors claim that the School of Proximity has provided a comprehensive picture and a systematic analysis of the dimensions, characteristics, and fields of application of the principle of proximity, and show that interest in proximity-related questions is the result of a long process through which scientists became aware of the value of this notion, and which has led to its gradual autonomization in the field of social sciences. Then are presented the main features of the School of Proximity, the story of the field of research, its theoretical variations, its ramifications, its conceptual stabilization and its inclusion in social sciences analysis. The authors give a canonical definition of proximity relationships by distinguishing between the two major polar categories - geographical and organized proximity - and identifying key achievements of this approach. They finally discuss the disciplinary approaches that are inspired nowadays by this approach, before opening the agenda of future research, in terms of analytical refinements and further extensions.

The first part of the Handbook is devoted to the major theoretical debates regarding the notion of proximity and to the construction of its analytical position in regional science.

Chapter 1, by Jean-Benoît Zimmermann, André Torre and Michel Grossetti, is entitled "The French School of Proximity: genesis and evolution of a school of thought". In this chapter, the authors revisit the origins and genesis of the French School of Proximity and its evolution through time, in order to better understand how and why the small group of researchers who were the driving force of this new way of thinking were quickly able to get a real legitimacy and effective recognition. First of all, it was clear that the role of space in economic dynamics was too often the subject of confusion and abusive assertions. Asking this question in terms of coordination made it possible to consider non-spatial factors in the analysis. The notion of proximity as a polysemic concept therefore opened the way to understanding how space matters or not, and together with these other factors thus a renewed approach of questions related to space and territories. But, even starting from issues of an economic nature, such an approach could not remain limited to its economic dimension, the questions of coordination involving social individuals, located in geographical space but also embedded in bundles of relationships and in institutions. Thus, it had to broaden very quickly to other disciplines in social sciences which largely contributed to consolidate the bases of what became a multidisciplinary approach and to develop theoretical as well as empirical tools. 
Chapter 2, by Pierre-Alexandre Balland, Ron Boschma and Koen Frenken, is entitled "Proximity, innovation and networks: a concise review and some next steps". In this chapter the authors make a review of proximity researches on collaborative innovation among organizations. They discuss the basic theorical tenets of these collaborations, summarize empirical findings on the roles of various forms of proximity, and assess the theoretical and empirical advances in proximity research based on the bilateral relationships rather than full networks. They do so by first discussing the basic premises and findings of proximity research in the area of knowledge production, innovation and networks, and turn to the aggregate level of analysis of inter-organizational relations within innovation systems. They end with a discussion of some of the next steps that can be taken in proximity research as applied to collaborative innovation, highlighting the need to understand proximity dynamics in addition to statics and the need to move beyond bilateral relations by looking at organizations' portfolio of relations.

Chapter 3, by Maryline Filippi, André Torre, Etienne Polge and Frederic Wallet, is entitled "The School of Proximity, genesis and development of a scientific notion". The authors analyze the complex notion of proximity regarding the "Proximity School" as a knowledge community in order to better understand how this concept becomes so used for territorial development processes, innovation analysis and public policy. Mobilizing an original publications' database (1990-2015), they apply co-authorship network analysis with centrality, density and transitivity measures. The results help to identify three main periods of development: birth and emergence, statement, maturity and dissemination. The evolution of this knowledge community allows us to understand how knowledge building process and social rules' changes occurred in the different periods. From the informal group to a well-known school, the authors have created step by step an analytical tool used by economists, geographers, planners, sociologists, regional scientists and others. But with the international consecration of the notion of proximity, the eponym group spread, victim of its success and with a loss of embeddedness.

Chapter 4, by Robert J. Stimson, is entitled "Proximity and regional development: an overview". It is based on the idea that geographical proximity is a core concept in various disciplines and moreover in regional science. Proximity relates to agglomeration and clustering of activities and network linkages between firms. Proximity effects are playing a significant role, especially in the context of endogenous forces in regional development. The chapter discusses proximity effects and proximity relations and how the notion of proximity is embedded within the literature on industrial clusters, knowledge spillovers, innovation, the learning region, and regional innovation systems. The importance of proximity effects for consideration in regional policy and regional development practice is also discussed.

Chapter 5, by Bastien Bernela, Marie Ferru and Alain Rallet, is entitled "The impact of digital technologies on perceptions of proximity". This chapter searches to better understand how the growing development of digital technologies affects the perception of geographical proximity, a crucial issue to understand actors' behavior and socioeconomic coordination. Modern digital technologies modify the relationship to distance obviously, but also constraints related to face-to-face interactions, as well as the effects of non-geographical proximity. They modify actors' relationship not only to space but also to time: the continuous connection allowed by mobile technologies invites us to 
consider a new form of proximity that we call proximity on the move. We define this form of proximity in a spatio-temporal and dynamic way. Proximity on the move questions the ability of actors to coordinate in real time. It leads to a coupling between physical concentration and decentralization and the multiplication of temporary occupation sites. Proximity on the move makes a kind of co-presence possible while the parties involved are physically distant: mobility is no longer a tool to bring temporary actors together, but it becomes a dynamic environment for activity. It makes it possible to consider the "deformations" of proximities as actors move and appears as a promising avenue of research in the future.

The second part of the Handbook is devoted to the methods and analytical tools used to define and to analyze the various types of proximity.

Chapter 6, by Andrea Caragliu, is entitled "Measuring the unmeasurable: the empirics of non-geographical proximity". This chapter summarizes the empirical literature on the impact of non-geographical proximity on economic interactions. Two main criteria for classifying this literature are identified in the text: the way different types of nongeographical proximity influence different economic outcomes; and the recent turn of this literature from the simultaneous role of geographic and non-geographical proximity to empirical studies tackling the effect of pure non-geographical proximity. On the basis of these arguments, policy directions and directions for future research are discussed by the author.

Chapter 7, by John Aldo Lee, Cyril de Bodt, Ludovic Journaux and Lucile Sautot, is entitled "Proximities in dimensionality reduction". The objective of this chapter is to provide an overview of some classical and more recent methods of dimensionality reduction, to shed some light on them from the perspective of analyzing proximities, and to illustrate them with multivariate data that could be typically encountered in social sciences. The authors show that dimensionality reduction aims at representing highdimensional data in a lower-dimensional representation, while preserving their structure (clusters, outliers, manifold). It can be used for exploratory data visualization, data compression, or as a preprocessing to some other analysis in order to alleviate the curse of dimensionality. Data structure is usually quantified with indicators, like covariance between variables, or pairwise proximity relationships, like scalar products, distances, similarities, or neighborhoods. Complementary aspects like quality assessment and alternative metrics are also briefly developed in this chapter.

Chapter 8, by Marina Bertoncin and Andrea Pase is entitled "Geographical proximity questioned". It examines the different conceptions of space and distance that have evolved from geographical thought. The measurement and representation of different types of distance are varied and distinct. Following this theoretical line, the authors identify three spatial proximities (absolute, relative and relational) that can be useful while analyzing concrete territorial results. The varying conditions, which draw on different situations that geographers can observe and study on the ground, are driven by the presence or absence of the three categories of spatial proximity. In particular, the spatial proximities play a significant role in the construction and identification of places in a globalized world. By implementing this approach to the geographical aspects of proximity relations, a new alliance is formed between human geography and proximity studies, reintegrating the issue of proximity back to areas such as social geography, political geography, and historical geography. 
Chapter 9, by Céline Rozenblat, is entitled “Cities' systems and networks' proximities: toward a multiplex approach". This chapter discusses the deep co-constructive relations between cities and proximities through networks, in a multi-dimensional and multi-level perspective. The proposed "multiplex" framework combines the multi-dimensional contribution of Evolutionary Economic Geography with the multi-level theory of evolutionary cities' systems. This combination highlights the properties of the evolutive levels of organization (meso and macro) in multiple dimensions of networks, and describes how the induced proximities interact between these dimensions. The meso levels, where proximities are higher, create a high potential of networks' development and retention. In addition, the interactions between levels constitute an essential factor of dynamics. The diffusion processes occurring between levels and between dimensions define adaptive cycles which evolve in interaction all together. The multiplex proximity framework, shaping entangled cycles of dimensions and levels, is useful to better understand and model cross-level and cross-dimensional policies in order to support adaptation of cities' economies to global change.

The third part of the Handbook is devoted to the questions of innovation and knowledge, which gave birth to many studies in the field of the analysis of proximity relations.

Chapter 10, by Riccardo Crescenzi, Max Nathan and Andrés Rodríguez-Pose, is entitled "Proximity and collaborative knowledge creation". In this chapter the authors examine the collaborations between inventors in the United Kingdom (UK) by looking at what types of proximities - geographical, organizational, cognitive, social, and cultural-ethnic - between inventors are prevalent in partnerships that ultimately lead to technological progress. The chapter explores the link between all these proximities and co-patenting by multi-patent inventors. The results show that organizational proximity is an essential characteristic of co-patenting teams together with social proximity formed by means of previous collaborations. Multiple patent inventors tend to patent much more with other inventors in close organizational proximity and within established social networks with space mediating the effect of all types of proximities considered. This implies that ideas flow inside very well-structured organizational and social pipelines. Nevertheless, the functioning of these communication channels improves in close geographical proximity.

Chapter 11, by Philip Cooke, is entitled "Fluctuating proximities and clusters: the future is already here - it's just not very evenly distributed". This chapter reviews three epochs of economic geography related to proximity in clusters, starting with Marshallian industrial districts. The periodization is followed by the overtaking of that spatial concentration model and concept in favor of a "structural" model configuration based on spatial divisions of labor. This, in turn, was displaced by the preference for observing and analyzing the "clusters" of geographical proximity. It is now being re-theorized in terms of a new "digital" model of industry organization based on machine learning "regions" that have evolved "cluster-platforms" in knowledge intensive 4.0 Web locations that combine cognitive, corporate and global connectivity with talent-intensive, entrepreneurial, and local geographical proximity. This latest form of economic geography has exploited neoliberal deregulation, robotization, and "big data" mastery over increasingly docile and precarious labor in an organizational mode that has replaced classic "Fordism" and its successor "Toyotism" with a variant of "Uberization" mediated only by money. 
Chapter 12, by Nicola Cortinovis and Frank van Oort, is entitled "Economic networks, innovation and proximity". The authors argue that despite the identification of various forms of proximity impacting on localized innovation and economic growth, claims on certain proximity types dominating over others have to be treated with caution. Besides physical proximity, crucial for face-to-face contact and tacit knowledge exchange, economic network proximity is argued to be important for the distribution and application of novel ideas. Such networks, like research cooperation, trade- and investment-related global networks, and labor market-related networks of mobility, migration and skill-relatedness, are empirically shown to be complementary to physical proximity. Because of equivalence in network structures and outcomes (many forms of proximity overlap with each other), identifying dominance of proximity types is difficult. The authors advocate that given the identified heterogeneity and complexity of direct and indirect relationships between economic networks, innovation and localized and clustered growth, this makes regional policy a much more complex task.

Chapter 13, by Marianne Steinmo and Thomas Lauvås, is entitled "The role of proximity dimensions in university-industry collaboration: a review and research agenda". The authors argue that university-industry collaboration is an important external source of knowledge in the development of innovations across a wide range of industries. Although many firms recognize the importance of collaborating with universities in innovation development, the collaboration process may be challenging due to the opposing logics of academic publications and industrial innovations. To overcome potential collaboration challenges, the proximity literature indicates that different dimensions of proximity facilitate beneficial interactions between firms and universities and increase learning and innovation. Hence, in this chapter, the authors examine the empirical evidence that is available on proximity dimensions in university-industry collaboration aiming at innovation. As such, they review prior research on geographical, cognitive, organizational, social and technological proximity in university-industry collaboration and discuss research gaps and avenues for future studies.

Chapter 14, by Rolf Sternberg, is entitled "Entrepreneurship and proximity". This chapter investigates whether and how various dimensions of proximity play a role for early-stage entrepreneurship. In order to explain the mechanisms and still keep the overview character of this short chapter it distinguishes between nascent entrepreneurs (pre-entry stage) and new entrepreneurs (post-entry stage) on the one hand and between geographical, social, institutional, cognitive, and organizational proximity on the other. The relevance of selected proximity dimensions for early-stage entrepreneurship differs significantly depending on the stage of entrepreneurship, but also on the sometimes very specific conditions in particular places. This does not mean that geographical proximity is always more important than the other four dimensions. In particular, social and cognitive proximity also often play a crucial role for the decision to start a firm as well as for its growth. This chapter also reports a complex system of interplays between several of the five dimensions when it comes to their impact on early-stage entrepreneurship. Empirical studies by economists, sociologists, psychologists, and management scholars in particular show that these five proximity dimensions rarely exhibit an isolated impact on early-stage entrepreneurship but are usually intertwined in complex interdependencies, the detailed empirical relevance of which has still to be unraveled by future research. 
Chapter 15, by Harald Bathelt, Pengfei Li and Yi-wen Zhu, is entitled "Geographies of temporary markets: an anatomy of the Canton Fair". In this chapter on temporary proximity, the authors underline that while recent research on temporary clusters and temporary markets has emphasized the knowledge generation processes associated with trade fairs, little is known about the knowledge exchanges that are embedded in market relations at these events. They use the case of the Canton Fair in Guangzhou, the largest trade fair in China, to illustrate that such events do not operate as a single market but that they generate multiple dynamic market configurations, which entail different flows of knowledge, goods and people. Four types of market configurations are identified that simultaneously develop at this event. The findings contribute to a more comprehensive understanding of the dynamics of market relations, knowledge and transactions in temporary spatial settings.

The last part of the Handbook is devoted to the emerging issues and to the new challenges and topics faced by the proximity analysis.

Chapter 16, by Patrick Mundler, is entitled "The role of proximity in food systems". The author refers to proximity food supply chains, which, through their organizational structure, attempt to reduce the distances between consumers and what they eat by mobilizing the geographic and relational proximities that exist among stakeholders. The concept of distance is multifaceted. It can refer to the growing physical distance in kilometers that food travels before reaching consumers, a trend that has disconnected the act of eating from the socio-cultural and geographic context in which food is grown. As a notion, distance can also be socio-economic in nature, as reflected in the lengthening of supply chains and the rising phenomenon of stakeholder specialization. Finally, it can imply a cognitive separation, fueled by the increased focus on food processing, which hinders the ability of consumers to feel a direct link with the original product and the natural resources used to produce it. Although proximity food supply chains lead to a spatial and relational rapprochement between farmers and consumers, these forms of proximity are not neutral and must be activated as resources if they are to contribute to the development of such networks. As resources, different proximities can complement and sometimes even compensate for each other, although they can also become constraining factors.

Chapter 17, by Christophe Beaurain and Sabrina Dermine-Brullot, is entitled "Industrial and territorial ecology: what types of proximity at work?" For several years, industrial ecology highlights the importance of interactions and industrial synergies in setting up a circular functioning of the economy, less intensive in consuming raw materials and energy and producing less waste, and is interested in the question of the location of these flows and the importance of actors' behaviors. In this chapter, the authors highlight the contribution of proximity theory to an analysis of the territorial dimension of these synergies located at the heart of industrial ecological approaches. They first underline the useful insight it provides on the role of geographical proximity and its various manifestations, and the establishment of these flows. Second, they emphasize the relevance of mobilizing the relational dimension of proximity to understand the mechanisms linked to the emergence and maintenance over time of collective action, on which an industrial ecology approach is based.

Chapter 18, by Habibullah Magsi and Muazzam Sabir, is entitled "Infrastructural development projects and proximity analysis". It explores the proximity relations in 
the dynamics of stakeholders' networks of opponents to infrastructure projects, in the cases of Diamer Basha and Chotiari dams in Pakistan. Both cases are studied in terms of superposition of land uses and their consequences. The results show that the authorities have disregarded the local populations' rights while constructing development projects in the country. The authors claim that mobilization of different proximities could have improved the governance capacity in order to build prevention process, and particularly capacity for strong negotiations between main stakeholders to sign commitments for sustainable and equitable natural resource use and ecosystem management.

Chapter 19, by Karima Kourtit, Peter Nijkamp and Peter Elmlund, is entitled "Neighbourhood proximity: a microcosmic resilience perspective on cities". It is based on the idea that we observe nowadays a worldwide interest in the socio-economic significance of cities in a global network society. The question whether cities provide an attractive and resilient home base for people in the "New Urban World" draws increasing attention and calls for a thorough reflection on the essence of a city. The authors offer food for thought on the question: what makes the city a city? After an overview of arguments from the literature, they posit that cities are complex multi-layered organisms which provide a broad portfolio of services and functions to residents, visitors and the business sector. A city can be decomposed into disjoint, but geographically connected spatial units (e.g., neighborhoods, streets), which altogether form an interactive dynamic and resilient organism. They advocate a microcosmic perspective on city life, in which communities, neighborhoods and related social-cultural proximity patterns among people or agents in a locality play a key role for urban cohesion. Common public spaces in urban districts or neighborhoods find their origin in this shared spatial proximity.

Chapter 20, by Lise Bourdeau-Lepage, is entitled "The city: a question of proximity(ies)". This chapter aims at presenting the way in which the French School of Proximity has taken an interest in urban space. The author explains that the combination of temporary, permanent, virtual and organized geographical proximity enables the city to be the place par excellence for internal and external interactions, whether face-to-face or via ICT. Then she presents the state of research on the negative effects of the different types of proximity and/or a combination of several of types of proximity, in the city. Thus are underlined the loss of altruism linked to environmental overload, the disconnection of time and space with virtual overload or the production of entre-soi with social overload.

The general conclusion of the Handbook, by Roberta Capello, is entitled "The concept of proximity in regional science - a synthesis and future research avenues". Its aim is to highlight the main messages the reader can grasp by reading the Handbook, and what remains to be interpreted in this fascinating concept. This aim is achieved through a diachronic review of the nexus between space and proximity developed in the different regional growth theories, a nexus that evolves according to the economic advantages that proximity is called to explain. The author then underlines the fascinating role played by proximities in linking long distance networks and local space, that emerges from reading the Handbook, and concludes with a personal view on what remains to be studied. 
André Torre and Delphine Gallaud - 9781786434784 Downloaded from PubFactory at 04/26/2023 02:19:25AM via free access 